\title{
Leucocyte esterase dip-stick test as a point- of-care diagnostic for urogenital chlamydia in male patients: A multi-center evaluation in two STI outpatient clinics in Paramaribo and Amsterdam
}

\author{
Menne Bartelsman ${ }^{1 *}$ D, Henry J. C. de Vries ${ }^{1,2,3}$, Maarten F. Schim van der Loeff ${ }^{2,4}$, Leslie O. A. Sabajo ${ }^{5}$ \\ and Jannie J. van der Helm ${ }^{1,4}$
}

\begin{abstract}
Background: Point-of-care (POC) tests are an important strategy to address the epidemic of sexually transmitted infections (STIS). The leucocyte esterase test (LET) can be used as a POC test for chlamydia. The aim of this study was to determine the diagnostic accuracy of the LET to detect urogenital chlamydia among men at STI clinics in Paramaribo, Suriname and Amsterdam, the Netherlands.

Methods: Recruitment of patients took place in 2008-2010 in Suriname and in 2009-2010 in the Netherlands. Urine of patients was examined with the LET. The reference test was a nucleic acid amplification test (NAAT).

Results: We included 412 patients in Suriname and 645 in the Netherlands. Prevalence of chlamydia in Suriname and the Netherlands was respectively 22.8 and $13.6 \%$. The sensitivity of the LET was $92.6 \%(95 \% \mathrm{Cl}=85.3-97.0)$ and 77. $3 \%(95 \% \mathrm{Cl}=67.1-85.5)$ respectively, the specificity was $38.1 \%(95 \% \mathrm{Cl}=32.7-43.6 \%)$ and $58.1 \%(95 \% \mathrm{Cl}=53.9-62.3)$ respectively. The positive predictive value was $30.6 \%(95 \% \mathrm{Cl}=27.3-36.4)$ and $22.6 \%(95 \% \mathrm{Cl}=18.0-27.7)$ respectively and the negative predictive value was $94.5 \%(95 \% \mathrm{Cl}=89.1-97.8)$ and $94.2 \%(95 \% \mathrm{Cl}=91.1-96.4)$ respectively. The kappa was respectively 0.179 and 0.176 .

Conclusions: To diagnose urogenital chlamydia in men the LET performs poorly. It has a high negative but low positive predictive value. If the LET result is negative, chlamydia is accurately excluded, yet a positive result has a low predictive value. Whether the advantages of direct management based on LET outweigh the disadvantages of overtreatment is a subject for further studies.
\end{abstract}

Keywords: Sensitivity, Specificity, Chlamydia, Point-of-care test, Leucocyte esterase test, Chlamydia trachomatis

\section{Background}

Chlamydia trachomatis $(\mathrm{Ct})$ is the most common cause of nongonoccal urethritis in men [1]. In resource-limited settings management of male urethritis is generally based on a syndromic approach: when a patient has typical symptoms like urethral discharge, penile itching or

\footnotetext{
* Correspondence: mbartelsman@ggd.amsterdam.nl

${ }^{1}$ Department of Infectious Diseases, STI Outpatient Clinic, Public Health

Service of Amsterdam (GGD Amsterdam), Weesperplein 1, 1018, XA,

Amsterdam, The Netherlands

Full list of author information is available at the end of the article
}

dysuria, immediate treatment with antibiotics is given. Yet, with a syndromic approach, a significant proportion of men infected with $\mathrm{Ct}$ are missed, as the majority of men does not have symptoms [2]. The proportion of cases that are asymptomatic varies by population and ranges from 40 to $96 \%$ [3-5]. Moreover, a syndromic approach also can lead to overtreatment, antibiotic overconsumption, induction of side effects and antimicrobial resistance of infections like Neisseria gonorrhoeae $(\mathrm{Ng})$. Also, effective contact tracing and treatment is challenging based on a presumptive diagnosis only. 
Point of care (POC) management can partly overcome the disadvantages of syndromic management. POC testing is defined as medical testing at or near the site of patient care [6]. Ideally a POC test should meet the ASSURED criteria of the World Health Organization; Affordable, Sensitive, Specific, User-friendly, Rapid and Robust, Equipment-free, Deliverable to those who need them [7]. Recently, several companies have developed commercial POC tests based on bacterial antigens that provide rapid results for the detection of $\mathrm{Ct}$, but the sensitivity of these tests is low (17-65\%) and this precludes more widespread use in clinical settings [6, 8-10]. The Cepheid GeneXpert $\mathrm{Ct} / \mathrm{Ng}$ assay is a rapid $(<2 \mathrm{~h}$ to results) nucleic acid amplification test (NAAT) assay that can be performed in on-site laboratories, but the high costs are an obstacle for implementation in low-and middle-income countries. Moreover it is questionable if patients are willing to wait $2 \mathrm{~h}$ for their test result [11]. As long as no other promising POC test is available, sexually transmitted infections (STI) clinics are dependent on convential techniques like light microscopy and dipstick urinalysis with the leucocyte esterase test (LET).

Light microscopy is used in the POC management of various STIs [1]. However, light microscopy requires a laboratory infrastructure that is often lacking in resourcelimited settings. In these settings the LET might be an alternative for light microscopy, because it is cheap, easy to perform and does not require a laboratory [12, 13]. LET is available as dipstick assay and is used to detect a urinary inflammatory reaction through the presence of an esterase enzyme produced by polymorph nuclear leucocytes (PMNL) in the urine, possibly associated with either a urinary tract infection or STI in males [14].

Suriname is a middle-income country in South America with most of the population concentrated in the coastal region, and the remainder in sparsely populated, predominantly remote areas. For these settings, an equipmentfree POC test could be of great benefit. The Netherlands is a high-income country in Europe, but also there an equipment-free POC test may be useful in certain primary health care settings like general practitioners clinics.

Previous studies that investigated the performance of the LET to detect urogenital $\mathrm{Ct}$ in males have reported sensitivities and specificities ranging from 46 to $100 \%$ and 60 to $96 \%$ respectively [15-19]. The variety of outcomes can be explained by a difference in settings, patient groups, experimental test thresholds and reference tests. In most of the studies obsolete reference tests like ligase chain reaction (LCR) were used and/or investigated the LET in either symptomatic or asymptomatic patients in one setting [16, 20-28].

The aim of our study was to evaluate the diagnostic performance of the LET as a POC test for urogenital Ct compared to NAAT in male patients irrespective of symptoms attending STI outpatient clinics in two different settings; an outpatient clinic in a middle-income country, Suriname, and in a high-income country, the Netherlands.

\section{Methods \\ Study design, selection of patients \\ Participants were recruited at:}

1. The Dermatological Service in Paramaribo, an integrated outpatient clinic that offers free-of-charge examination and treatment of STIs and infectious skin diseases such as leprosy and leishmaniasis. Recruitment took place between March 2008 and July 2010.

2. The STI Outpatient Clinic of the Public Health Service of Amsterdam, which is a low threshold clinic serving over 40,000 patients annually. Patients were prioritized based on a short questionnaire to estimate the risk of having an STI, as described before [29]. The following categories were regarded as high risk: age $<25$ years, men who have sex with men, born in an STI and HIV endemic country, receiving money/goods for sex, paying for sex, $\geq 3$ partners in the previous 6 months, reporting a sexual partner with a partner born in an STI and HIV endemic country, being notified by a sex partner or having STI related symptoms. Patients regarded as high-risk were eligible to participate; recruitment took place between November 2009 and May 2010.

In both countries demographic characteristics and data regarding symptoms were obtained using a questionnaire. Patients were regarded as symptomatic when either dysuria, urethral discharge or scrotal pain was reported in the questionnaire.

\section{Leucocyte esterase test (LET)}

The LET (Combur ${ }^{2}$ Test LN, Roche Diagnostics, GmbH, Mannheim, Germany) is a non-specific rapid dipstick assay used to detect the presence of an esterase enzyme produced by PMNL's in urine. The presence of PMNL's in urine indicates an inflammatory response, possibly caused by an infection. In Suriname and the Netherlands patients provided urine samples that were immediately brought to the on-site laboratory by nurses. A laboratory technician performed the LET within two hours of sample collection and placed the dipstick in a urine sample according to the manufacturer's instructions. The results were read after 2 and $5 \mathrm{~min}$. Using a reference color table, the dipstick differentiated between negative, 1+ (ca. 10-25 PMNL/ $\mu \mathrm{l}$ ), 2+ (ca. $75 \mathrm{PMNL} / \mu \mathrm{l}$ ), 3+ (ca. $500 \mathrm{PMNL} /$ $\mu \mathrm{l})$. The LET was regarded positive when the dipstick colored $\geq 1+$. Patients with a failed or missing LET were excluded from the analyses. 


\section{Reference tests}

The Aptima Ct assay (Hologic Gen-Probe, USA) was used as the reference NAAT to detect urogenital Ct both in the Netherlands and Suriname. Reference testing was performed on the same urine sample that was used for the LET. In the Netherlands the samples were tested the same week at the Public Health Laboratory in Amsterdam. In Suriname, the samples were collected according to the manufacturer's (Hologic) instructions, stored in a fridge (at temperature between $2^{\circ}$ and $7{ }^{\circ} \mathrm{C}$ ) and packed according to international air transport association (IATA) rules for transport by plane to the Public Health Laboratory in Amsterdam for NAAT testing. According to the manufacturer's instructions the samples had to be analysed within 30 days after collection. In our study 349 of the 412 samples $(84.7 \%)$ were analysed within 30 days and 63 samples $(15.3 \%)$ were tested between 31 and 48 days (median time: 35 days (IQR 33-36 days)). More details about the data collection were previously described [8, 30].

\section{Diagnostic performance and statistical analysis}

The diagnostic performance of the LET was investigated by calculating the sensitivity, specificity, positive predictive value (PPV), negative predictive value (NPV), the percentage agreement and kappa. A kappa value $>0.61$ was considered as good test agreement, $\kappa=0.41-0.60$ as fair agreement, $\mathrm{K}=0.21-0.40$ as slight agreement, $\mathrm{K}=0.01-0.20$ as poor agreement, and $\kappa \leq 0.00$ as no agreement) [31].

As prescribed by the manufacturer the LET was read 2 min after it was placed in urine (from here onwards described as "LET $2 \mathrm{~m}$ "). We also read the result of the LET 5 min after it was placed in urine (from now on described as "LET $5 \mathrm{~m}$ ") to evaluate the influence of timing on the diagnostic performance.

We compared the diagnostic performance of the LET $2 \mathrm{~m}$ between symptomatic and asymptomatic patients and conducted a subgroup analysis for individuals $<25$ years.

Characteristics of the study populations were compared using the Pearson's chi-square test. When the expected cell-count was $<5$ the Fisher's exact test was used. We considered a $p$-value $<0.05$ as statistically significant. Analyses were performed with SPSS package version 21 (SPSS Inc., Chicago, Illinois, USA) and STATA software V11.2 (Stata Corp, College Station, Texas, USA).

\section{Results}

\section{Study populations}

Suriname

In total 415 patients provided samples for the study, of which three patients had an invalid LET result and were excluded. From the remaining 412 patients 279 (67.7\%) of the patients were symptomatic and 133 (32.3\%) were asymptomatic. The median age was 28 years (interquartile range (IQR) 24.0-37.0). Ct prevalence was $22.8 \%$ (95\% CI 19.0-27.1) (Table 1).

\section{The Netherlands}

In total 647 patients provided samples for the study of which two patients had an invalid LET result and were excluded. Data regarding symptoms were missing for nine patients. Among the remaining 636 patients 257 (40.4\%) of the patients were symptomatic and 379 (59.6\%) were asymptomatic. The median age was 34 years (IQR 26.543.0). Ct prevalence was $13.6 \%$ (95 \% CI 11.2-16.5).

Table 1 Characteristics of male patients included at the Dermatological Service Paramaribo, Suriname, 2008-2010 and the STI Outpatient Clinic, Amsterdam, The Netherlands, 2009-2010

\begin{tabular}{|c|c|c|c|}
\hline & $\begin{array}{l}\text { Suriname } \\
(N=412)\end{array}$ & $\begin{array}{l}\text { The Netherlands } \\
(N=645)\end{array}$ & $P$ value \\
\hline Demographic characteristics & $n(\%)$ & $n(\%)$ & \\
\hline Median age (years) (IQR) & $28.0(24.0-37.0)$ & $34.0(26.5-43.0)$ & $<0.001$ \\
\hline Age (years) & & & $<0.001$ \\
\hline$<25$ & $109(26.5)$ & $110(17.1)$ & \\
\hline $25-29$ & $115(27.9)$ & $123(19.1)$ & \\
\hline $30-34$ & $59(14.3)$ & $108(16.7)$ & \\
\hline$\geq 35$ & $129(31.3)$ & $304(47.1)$ & \\
\hline Symptoms* & & & $<0.001$ \\
\hline No & $133(32.3)$ & $379(59.6)$ & \\
\hline Yes & $279(67.7)$ & $257(40.4)$ & \\
\hline HIV status ${ }^{* *}$ & & & $<0.001$ \\
\hline Negative & $392(95.1)$ & $519(80.5)$ & \\
\hline Positive & $2(0.49)$ & $113(17.5)$ & \\
\hline Unknown & $18(4.4)$ & $13(2.0)$ & \\
\hline Sexual preference* & & & $<0.001$ \\
\hline Heterosexual men & $402(98.3)$ & $282(43.7)$ & \\
\hline $\begin{array}{l}\text { Men who have sex } \\
\text { with men }\end{array}$ & $7(1.7)$ & $363(56.3)$ & \\
\hline \multicolumn{4}{|l|}{ Ct prevalence } \\
\hline Total population & $94(22.8)$ & 88 (13.6) & $<0.001$ \\
\hline$<25$ years & $29(26.6)$ & $21(19.1)$ & 0.185 \\
\hline Symptomatic patients* & $77(27.6)$ & $53(20.6)$ & 0.060 \\
\hline Asymptomatic patients* & $17(12.8)$ & $34(9.0)$ & 0.207 \\
\hline
\end{tabular}

Abbreviations: STI sexual transmitted infections; IQR interquartile range; $C t$ Chlamydia trachomatis

*Numbers do not add up to column total due to missing data; data on symptoms were missing for 9 patients in the Netherlands and data on sexual preference were missing for 3 patients in Suriname. Percentages were calculated based on those with non-missing data and thus add up to $100 \%$

**In the Netherlands a positive HIV status was based on a positive HIV test in the past or on the date of inclusion (noted in the patient records); a negative HIV status was based on a negative HIV test result on the date of inclusion. The HIV status was unknown when a HIV test was lacking on the date of inclusion. In Suriname the HIV status was self-reported at the date of inclusion 


\section{Ct prevalence and symptomatology in Suriname and the Netherlands}

In Suriname significantly more patients reported symptoms compared with the Netherlands; $67.7 \%$ (279/412) versus $40.4 \%(257 / 636)(p<0.001)$. Also a significantly higher prevalence of $\mathrm{Ct}$ was found in Suriname than in the Netherlands; $22.8 \%(94 / 412)$ versus $13.6 \%(88 / 645)$ $(p<0.001)$.

\section{Diagnostic performance \\ Suriname}

With LET $2 \mathrm{~m} \mathrm{284/412}$ had a positive and 128/412 a negative result. The LET $2 \mathrm{~m}$ showed a sensitivity of $92.6 \%$ (95 \% CI 85.3-97.0), a specificity of $38.1 \%$ (95\% CI 32.7-43.6 \%), a PPV of $30.6 \%$ (95 \% CI 27.3-36.4), an NPV of $94.5 \%$ (95\% CI 89.1-97.8) and a kappa of 0.179 (Table 2). The LET $5 \mathrm{~m}$ had a comparable sensitivity of $96.8 \%(p=0.1936)$ but a significantly lower specificity of $16.7 \%(p<0.001)$. The LET $2 \mathrm{~m}$ had a comparable sensitivity among symptomatic and asymptomatic patients, $92.2 \%$ versus $94.1 \%(p=0.786)$, but the specificity was significantly lower among the symptomatic patients compared with the asymptomatic patients; $30.2 \%$ versus $51.7 \%(p<0.001)$.

Among patients $<25$ years of age the sensitivity was $100 \%$ (95\% CI 90.2-100) for the LET 2 m, although this was not significantly different compared with the sensitivity of the those $\geq 25$ years; $89.2 \%$ (95 \% CI 79.195.6) $(p=0.066)$. Also the specificity was not significantly different; $41.3 \%$ (95 \% CI 30.9-52.3) versus $37.0 \%$ (95\% CI 30.8-43.4) $(p=0.496)$.

\section{The Netherlands}

With LET $2 \mathrm{~m} \mathrm{301/645}$ had a positive result and 344/ 645 had a negative result. The LET $2 \mathrm{~m}$ had a sensitivity of $77.3 \%$ (95 \% CI 67.1-85.5), a specificity of $58.1 \%$ (95 \% CI 53.9-62.3), a PPV of 22.6 \% (95 \% CI 18.0-27.7), an NPV of $94.2 \%$ (95\% CI 91.1-96.4) and a kappa of 0.176 (Table 3). The sensitivity of the LET $5 \mathrm{~m}$ was $90.9 \%$; significantly higher than the sensitivity of the LET $2 \mathrm{~m}(p=0.013)$. The specificity of the LET $5 \mathrm{~m}$ was $40.8 \%$; significantly lower than the specificity of the LET $2 \mathrm{~m}(p<0.001)$.

The sensitivity of the LET $2 \mathrm{~m}$ was not significantly different between symptomatic and asymptomatic patients $(p=0.096)$, but the specificity was significantly lower among symptomatic patients (51.0 \%) than among asymptomatic patients $(62.6 \%)(p=0.008)$. In the younger age group ( $<25$ years) a high prevalence of $\mathrm{Ct}$ was found (19.1\%) but the sensitivity and specificity of the LET $2 \mathrm{~m}$ in this group were not significantly different compared with the age group $\geq 25$ years ( $p=0.290$ and $p=0.377$ ).

Compared with Suriname the sensitivity of the LET $2 \mathrm{~m}$ was significantly lower; $77.3 \%$ versus $92.6 \%(p=0.004)$.

\section{Discussion}

To our knowledge this is the first multi-center study that evaluated the LET to detect urogenital Ct among men in clinics in both a middle-income country and in a highincome country with high Ct prevalences $(22.8 \%$ and $13.6 \%$, respectively).

Both in Suriname and the Netherlands the LET $2 \mathrm{~m}$ had a reasonably high sensitivity (respectively 92.6 and $77.3 \%$ ) but a poor specificity (respectively 38.1 and $58.1 \%$ ) and a poor agreement (respectively kappa 0.179 and 0.176). The significantly higher sensitivity of the LET $2 \mathrm{~m}$ we found in Suriname compared with the Netherlands can be caused by a difference in study populations. In the Netherlands significantly more patients were asymptomatic (59.6\%) compared with Suriname (32.3\%). Wiggins et al. suggested that asymptomatic patients have infections with a lower infection-load and therefore fewer leucocytes which might cause false negative test results [32]. The same group showed that the number of leucocytes (predominantly PMNL's) was strongly correlated with urethritis and proved that men with asymptomatic urethritis have fewer PMNL's than symptomatic men [33]. A Swedish study conducted among 480 male patients of an STI clinic (also with Ct as outcome and $\geq 1+$ as cutoff point) supports this hypothesis: the LET had a significantly higher sensitivity (69.6\% versus $25.9 \%$ ) but a lower specificity (76.3\% versus $85.8 \%$ among symptomatic patients compared with asymptomatic patients [17].

Moreover, in both countries the LET $2 \mathrm{~m}$ showed a significantly higher specificity in asymptomatic patients compared with symptomatic patients. A possible explanation can be that symptomatic patients had relatively more often infections like Neisseria gonorrhoeae $(\mathrm{Ng})$, Mycoplasma genitalum (Mg) or Trichomonas Vaginalis (Tv) compared to asymptomatic patients which could have caused a relative higher proportion of 'false positive' results [34-36]. Also in a previous study we showed that light microscopic examination of Gram stained urethral smears is less specific to detect urogenital $\mathrm{Ct}$ infections when done in samples of symptomatic males only [37].

In the Netherlands the sensitivity of the LET increased and in both countries the specificity decreased when the incubation time was prolonged from 2 to $5 \mathrm{~min}$. A study of Morré et al. found also a higher sensitivity when the reading time was prolonged from 2 to $5 \mathrm{~min}$ [38].

The strength of our study is that we explored the diagnostic accuracy of the LET among both symptomatic and asymptomatic male patients in a high- and in a middle-income country with a different $\mathrm{Ct}$ prevalence. A shortcoming is that we only focused on $\mathrm{Ct}$ and not on other causative micro-organisms of urethritis like $\mathrm{Ng}, \mathrm{Mg}$ or Tv. Evaluation of other micro-organisms could have 
Table 2 Diagnostic performance* of the Leucocyte Esterase Test (LET) after a reading time of 2 min ("LET 2 m") and 5 min ("LET 5 m") in the detection of urogenital Chlamydia Trachomatis (Ct) among male patients, Dermatological Service in Paramaribo, Suriname, 2008-2010

\begin{tabular}{|c|c|c|c|c|c|c|c|c|c|c|c|}
\hline & $\mathrm{N}$ & LET + NAAT+ & LET-NAAT+ & LET + NAAT- & LET- NAAT- & Sens. $(95 \%$ Cl) & Spec. $(95$ \% Cl) & PPV $(95 \%$ Cl) & NPV (95 \% Cl) & Agr. & Kappa \\
\hline \multicolumn{12}{|l|}{ LET $2 \mathrm{~m}$} \\
\hline ALL & 412 & 87 & 7 & 197 & 121 & $92.6 \%(85.3-97.0)$ & $38.1 \%(32.7-43.6)$ & $30.6 \%(27.3-36.4)$ & $94.5 \%$ (89.1-97.8) & $50.5 \%$ & 0.179 \\
\hline Sympto-matic patients & 279 & 71 & 6 & 141 & 61 & $92.2 \%(83.8-97.1)$ & $30.2 \%(24.0-37.0)$ & $33.5 \%(27.2-40.3)$ & $91.0 \%$ (81.5-96.6) & $47.3 \%$ & 0.145 \\
\hline Asympto-matic patients & 133 & 16 & 1 & 56 & 60 & $94.1 \%(71.3-99.9)$ & $51.7 \%(42.3-61.1)$ & $22.2 \%(13.8-32.9)$ & $98.4 \%(91.2-100)$ & $57.1 \%$ & 0.193 \\
\hline$<25$ years & 109 & 29 & 0 & 47 & 33 & $100 \%(88.1-100)$ & $41.3 \%$ (30.4-52.8) & $38.2 \%(27.2-50.0)$ & $100 \%(89.4-100)$ & $56.9 \%$ & 0.272 \\
\hline \multicolumn{12}{|l|}{ LET $5 \mathrm{~m}$} \\
\hline ALL & 412 & 91 & 3 & 265 & 53 & $96.8 \%(91.0-99.3)$ & $16.7 \%(12.7-21.2)$ & $25.6 \%(21.1-30.4)$ & $94.6 \%(85.1-98.9)$ & 35.0 & 0.068 \\
\hline
\end{tabular}

*Sensitivity (Sens.), Specificity (Spec.), Positive Predictive Value (PPV), Negative Predictive Value (NPV), Agreement (Agr.) and Kappa 
Table 3 Diagnostic performance* of the Leucocyte Esterase Test (LET) after a reading time of 2 min ("LET 2 m") and 5 min ("LET 5 m") in the detection of urogenital Chlamydia Trachomatis (Ct) among male patients, STI outpatient clinic, Amsterdam, The Netherlands, 2009-2010

\begin{tabular}{|c|c|c|c|c|c|c|c|c|c|c|c|}
\hline & $\mathrm{N}$ & LET+ NAAT+ & LET- NAAT+ & LET+ NAAT- & LET- NAAT- & Sens. (95 \% Cl) & Spec. $(95 \%$ Cl) & PPV $(95 \%$ Cl) & NPV (95 \% Cl) & Agr. & Kappa \\
\hline \multicolumn{12}{|l|}{ LET $2 \mathrm{~m}$} \\
\hline ALL & 645 & 68 & 20 & 233 & 324 & $77.3 \%(67.1-85.5)$ & $58.1 \%(53.9-62.3)$ & $22.6 \%(18.0-27.7)$ & $94.2 \%(91.1-96.4)$ & $60.8 \%$ & 0.176 \\
\hline Sympto-matic patients & 257 & 44 & 9 & 100 & 104 & $83.0 \%(70.2-91.9)$ & $51.0 \%(43.9-58.0)$ & $30.6 \%(23.2-38.8)$ & $92.0 \%$ (85.4-96.3) & $57.6 \%$ & 0.208 \\
\hline Asympto-matic patients & 379 & 23 & 11 & 129 & 216 & $67.6 \%(49.5-82.6)$ & $62.6 \%(57.2-67.7)$ & $15.1 \%(9.8-21.8)$ & $95.1 \%(91.4-97.5)$ & $63.1 \%$ & 0.026 \\
\hline$<25$ years & 110 & 18 & 3 & 41 & 48 & $85.7 \%$ (63.7-97.0) & $53.9 \%(43.0-64.6)$ & $30.5 \%(19.2-43.9)$ & $94.1 \%(84.8-98.8)$ & $60.0 \%$ & 0.068 \\
\hline \multicolumn{12}{|l|}{ LET $5 \mathrm{~m}$} \\
\hline ALL & 645 & 80 & 8 & 330 & 227 & $90.9 \%(82.9-96.0)$ & $40.8 \%(36.7-45.0)$ & $19.6 \%(15.9-23.8)$ & $96.6 \%$ (93.4-98.5) & $47.6 \%$ & 0.125 \\
\hline
\end{tabular}


been helped to interpret the difference of false positive results between symptomatic and asymptomatic patients. Another shortcoming is that not all NAAT samples from Suriname were analyzed within 30 days after collection (according to manufacturer's instructions) at the Public Health Laboratory in Amsterdam; 63 samples (15.3 \%) were analyzed between 31 and 48 days, although it is unlikely that this small delay has influenced the performance of the NAAT [39]. Recently, several companies have developed commercial POC tests that provide rapid results for the detection of $\mathrm{Ct}$, however, the sensitivity of these tests is low (25-65\%) and precludes more widespread use in clinical settings [8-10, 40, 41]. An exception of a POC test with a high sensitivity is the GeneXpert $\mathrm{Ct} / \mathrm{Ng}$ (Cepheid), a cartridge-based automated test that can identify $\mathrm{Ct}$ and $\mathrm{Ng}$ infections by NAAT within $2 \mathrm{~h}$, but the high costs hinder the implementation in low-and middle-income countries [42]. Moreover, not all patients may be willing to wait for the results for $2 \mathrm{~h}$. Earlier we showed that in the Surinamese setting only $26.7 \%$ of the female STI visitors would be willing to wait for their POC test results for more than one hour [8].

Syndromic management based on symptoms falls short and leaves many asymptomatic infections untreated $[40,43]$. As long as no accurate and affordable POC tests for $\mathrm{Ct}$ are available, the LET could be a cheap and an easy to perform alternative to exclude urogenital $\mathrm{Ct}$ infections among men in settings where any $\mathrm{Ct}$ diagnostics are lacking. The sensitivity we found for the LET in Suriname (91.6 \%) was reasonably high. It is estimated that a POC test of even moderate sensitivity (63\%) combined with immediate treatment on-site may lead to the treatment of more infected individuals than an ultra-sensitive and specific NAAT alone when patient return is low [44].

Settings lacking any STI laboratory diagnostics now mainly rely on syndromic management for STI treatment. Further studies into the diagnostic performance of the LET test as opposed to routine syndromic management are needed; the effect on overtreatment due to low specificity would be an important end point.

Our previous study on the cost-effectiveness of microscopic examination of Gram stained urethral smears compared to NAAT at the STI outpatient clinic in Amsterdam showed a sensitivity of $83.8 \%$ and specificity of $74.1 \%$, comparable with the sensitivity and specificity of the LET we found in the current study [37]. However, if available, microscopy is preferable above the LET because it can also detect $\mathrm{Ng}$ infections by finding Gram negative diplococci in a Gram stained smear.

\section{Conclusion}

In conclusion, we showed that the LET has a reasonably high sensitivity but a low specificity to diagnose urogenital $\mathrm{Ct}$ in male STI clinic visitors. Future studies that also include the detection of other main causative infections of urethritis are needed to compare the cost-effectiveness of the LET in comparison with syndromic management.

\section{Abbreviations}

ASSURED: Affordable, Sensitive, Specific, User-friendly, Rapid and Robust Equipment-free, Deliverable; Ct: Chlamydia trachomatis; IATA: International air transport association; IQR: Interquartile range; LCR: Ligase chain reaction;

LET: Leucocyte esterase test; Mg: Mycoplasma genitalum; NAAT: Nucleic acid amplification test; Ng: Neisseria gonorrhoeae; NPV: Negative predictive value; PMNL: Polymorph nuclear leucocytes; POC: Point of care; PPV: Positive predictive value; STI: Sexually transmitted infections; TV: Trichomonas Vaginalis

\section{Acknowledgements}

The authors would like to thank all nurses and laboratory technicians of the Dermatological Service in Paramaribo and the STI outpatient clinic in Amsterdam for data collection.

\section{Funding}

This study was financed by the Research and Development department of the Public Health Service of Amsterdam, no 2382.

\section{Availability of data and material}

Data are available upon request.

\section{Authors' contributions}

HJCdV, JJvdH and LOAS were responsible for the design of the study. JJvdH and LOAS collected the data in Suriname. JJvdH collected the data in the Netherlands. MB and JJvdH did the statistical analysis of the diagnostic outcomes. MB drafted the paper. MFSvdL advised on statistical analysis and interpretation. HJCdV supervised the overall study. All authors reviewed and approved the final article.

\section{Competing interests}

Maarten Schim van der Loeff and Henry de Vries served on an Advisory Board of GSK

\section{Consent for publication}

Not applicable.

\section{Ethics approval and consent to participate}

The study was approved by the ethics committee of the Ministry of Health of the Republic of Suriname (VG010-2007) and the ethics committee of the Academic Medical Center, University of Amsterdam, the Netherlands (MEC07/ 127). Patients participated anonymously and gave written informed consent.

\section{Author details}

${ }^{1}$ Department of Infectious Diseases, STI Outpatient Clinic, Public Health Service of Amsterdam (GGD Amsterdam), Weesperplein 1, 1018, XA, Amsterdam, The Netherlands. ${ }^{2}$ Center for Infection and Immunology Amsterdam (CINIMA), Academic Medical Center (AMC), University of Amsterdam, Amsterdam, The Netherlands. ${ }^{3}$ Department of Dermatology, Academic Medical Center (AMC), University of Amsterdam, Amsterdam, The Netherlands. ${ }^{4}$ Department of Research, Public Health Service of Amsterdam (GGD Amsterdam), Nieuwe Achtergracht 100, 1018, WT, Amsterdam, The Netherlands. ${ }^{5}$ Dermatological Service, Ministry of Health Suriname,

Tourtonnelaan 5, Paramaribo, Suriname.

Received: 6 July 2016 Accepted: 20 October 2016

Published online: 03 November 2016

\section{References}

1. Brill JR. Diagnosis and treatment of urethritis in men. Am Fam Physician. 2010;81:873-8.

2. $\quad$ Alary M, Lowndes $C M$, Mukenge-Tshibaka $L$, et al. Sexually transmitted infections in male clients of female sex workers in Benin: risk factors and reassessment of the leucocyte esterase dipstick for screening of urethral infections. Sex Transm Infect. 2003;79:388-92. 
3. Cecil JA, Howell MR, Tawes JJ, et al. Features of Chlamydia trachomatis and Neisseria gonorrhoeae infection in male Army recruits. J Infect Dis. 2001;184:1216-9.

4. Detels R, Green AM, Klausner JD, et al. The incidence and correlates of symptomatic and asymptomatic Chlamydia trachomatis and Neisseria gonorrhoeae infections in selected populations in five countries. Sex Transm Dis. 2011;38:503-9.

5. Kent CK, Chaw JK, Wong W, et al. Prevalence of rectal, urethral, and pharyngeal chlamydia and gonorrhea detected in 2 clinical settings among men who have sex with men: San Francisco, California, 2003. Clin Infect Dis. 2005;41:67-74.

6. Huppert J, Hesse E, Gaydos CA. What's the point? How Point-of-Care STI tests can impact infected patients. Point Care. 2010;9:36-46.

7. Peeling RW, Holmes KK, Mabey D, et al. Rapid tests for sexually transmitted infections (STIS): the way forward. Sex Transm Infect. 2006;82 Suppl 5:v1-6.

8. van der Helm JJ, Sabajo LO, Grunberg AW, et al. Point-of-care test for detection of urogenital chlamydia in women shows low sensitivity. A performance evaluation study in two clinics in Suriname. PLoS One. 2012;7:e32122.

9. van Dommelen $\mathrm{L}$, van Tiel FH, Ouburg $\mathrm{S}$, et al. Alarmingly poor performance in Chlamydia trachomatis point-of-care testing. Sex Transm Infect. 2010;86:355-9.

10. de Herbst CS, Bristow CC, Joseph DD, et al. A systematic review of point of care testing for Chlamydia trachomatis, Neisseria gonorrhoeae, and Trichomonas vaginalis. Infect Dis Obstet Gynecol. 2016;2016:4386127.

11. Gaydos CA, Van Der Pol B, Jett-Goheen M, et al. Performance of the Cepheid CT/NG Xpert Rapid PCR Test for Detection of Chlamydia trachomatis and Neisseria gonorrhoeae. J Clin Microbiol. 2013;51:1666-72.

12. Centers for Disease Control and Prevention. STD Treatment Guidelines 2015. Available at: https://www.cdc.gov/std/tg2015/.

13. Horner PJ, Blee K, Falk L, et al. 2016 European guideline on the management of non-gonococcal urethritis. Int J STD AIDS. 2016;27:928-37.

14. Rahman MS, Beever W, Skov S, et al. Using urinary leucocyte esterase tests as an indicator of infection with gonorrhoea or chlamydia in asymptomatic males in a primary health care setting. Int J STD AIDS. 2014;25:138-44.

15. Blake DR, Lemay CA, Gaydos CA, et al. Performance of urine leukocyte esterase in asymptomatic male youth: another look with nucleic acid amplification testing as the gold standard for Chlamydia detection. J Adolesc Health. 2005:36:337-41.

16. Sellors JW, Mahony JB, Pickard L, et al. Screening urine with a leukocyte esterase strip and subsequent chlamydial testing of asymptomatic men attending primary care practitioners. Sex Transm Dis. 1993;20:152-7.

17. Hedin G, Abrahamsson G, Dahlberg E. Urethritis associated with Chlamydia trachomatis: comparison of leukocyte esterase dipstick test of first-voided urine and methylene blue-stained urethral smear as predictors of chlamydial infection. APMIS. 2001;109:595-600.

18. Marrazzo JM, Whittington $\mathrm{WL}$, Celum $\mathrm{CL}$, et al. Urine-based screening for Chlamydia trachomatis in men attending sexually transmitted disease clinics. Sex Transm Dis. 2001;28:219-25.

19. Wood BJ, Gaydos JC, McKee Jr KT, et al. Comparison of the urine Leukocyte Esterase Test to a Nucleic Acid Amplification Test for screening non-health care-seeking male soldiers for Chlamydia trachomatis and Neisseria gonorrhoeae infections. Mil Med. 2007;172:770-2.

20. Bowden FJ. Reappraising the value of urine leukocyte esterase testing in the age of nucleic acid amplification. Sex Transm Dis. 1998;25:322-6.

21. Grosskurth H, Mayaud P, Mosha F, et al. Asymptomatic gonorrhoea and chlamydial infection in rural Tanzanian men. BMJ. 1996;312:277-80.

22. Jackson DJ, Rakwar JP, Chohan B, et al. Urethral infection in a workplace population of East African men: evaluation of strategies for screening and management. J Infect Dis. 1997;175:833-8.

23. Mayaud P, Changalucha J, Grosskurth $\mathrm{H}$, et al. The value of urine specimens in screening for male urethritis and its microbial aetiologies in Tanzania. Genitourin Med. 1992;68:361-5

24. O'Brien SF, Bell TA, Farrow JA. Use of a leukocyte esterase dipstick to detect Chlamydia trachomatis and Neisseria gonorrhoeae urethritis in asymptomatic adolescent male detainees. Am J Public Health. 1988;78:1583-4.

25. Patrick DM, Rekart ML, Knowles L. Unsatisfactory performance of the leukocyte esterase test of first voided urine for rapid diagnosis of urethritis. Genitourin Med. 1994;70:187-90

26. Shafer MA, Schachter J, Moscicki AB, et al. Urinary leukocyte esterase screening test for asymptomatic chlamydial and gonococcal infections in males. JAMA. 1989:262:2562-6.
27. Tyndall MW, Nasio J, Maitha G, et al. Leukocyte esterase urine strips for the screening of men with urethritis-use in developing countries. Genitourin Med. 1994;70:3-6.

28. Watson-Jones D, Mugeye $K$, Mayaud $P$, et al. High prevalence of trichomoniasis in rural men in Mwanza, Tanzania: results from a population based study. Sex Transm Infect. 2000;76:355-62.

29. Heijman TL, Van der Bij AK, de Vries HJ, et al. Effectiveness of a risk-based visitor-prioritizing system at a sexually transmitted infection outpatient clinic. Sex Transm Dis. 2007;34:508-12.

30. Bom RJ, van der Helm JJ, Bruisten SM, et al. The role of Surinamese migrants in the transmission of Chlamydia trachomatis between Paramaribo, Suriname and Amsterdam, The Netherlands. PLoS One. 2013:8:e77977.

31. Zweig MH, Campbell G. Receiver-operating characteristic (ROC) plots: a fundamental evaluation tool in clinical medicine. Clin Chem. 1993;39:561-77.

32. Wiggins $R$, Graf $S$, Low $N$, et al. Real-time quantitative PCR to determine chlamydial load in men and women in a community setting. J Clin Microbiol. 2009:47:1824-9.

33. Wiggins RC, Holmes $\mathrm{CH}$, Andersson M, et al. Quantifying leukocytes in first catch urine provides new insights into our understanding of symptomatic and asymptomatic urethritis. Int J STD AIDS. 2006;17:289-95.

34. Shahmanesh $\mathrm{M}$, Radcliffe KW. Is the urethral smear necessary in asymptomatic men attending a genitourinary medicine clinic? Sex Transm Infect. 2007:83:79-81.

35. Ross JD, Jensen JS. Mycoplasma genitalium as a sexually transmitted infection: implications for screening, testing, and treatment. Sex Transm Infect. 2006;82:269-71

36. Falk L, Fredlund $H$, Jensen JS. Symptomatic urethritis is more prevalent in men infected with Mycoplasma genitalium than with Chlamydia trachomatis. Sex Transm Infect. 2004:80:289-93.

37. Bartelsman M, van Rooijen MS, Alba S, et al. Point-of-care management of urogenital Chlamydia trachomatis via Gram-stained smear analysis in male high-risk patients. Diagnostic accuracy and cost-effectiveness before and after changing the screening indication at the STI Clinic in Amsterdam. Sex Transm Infect. 2015:91:479-84.

38. Morre SA, Spaargaren J, Veldhuijzen IK, et al. Evaluation of the leukocyte esterase test (LET) as pre-screening test to reduce costs for national population-based Chlamydia trachomatis screening programs. J Adolesc Health. 2006;38:332-3.

39. Boyadzhyan B, Yashina T, Yatabe JH, et al. Comparison of the APTIMA CT and GC assays with the APTIMA combo 2 assay, the Abbott LCX assay, and direct fluorescent-antibody and culture assays for detection of Chlamydia trachomatis and Neisseria gonorrhoeae. J Clin Microbiol. 2004;42:3089-93.

40. Natoli L, Maher L, Shephard M, et al. Point-of-care testing for chlamydia and gonorrhoea: implications for clinical practice. PLoS One. 2014;9:e100518.

41. Causer LM, Hengel B, Natoli L et al. A field evaluation of a new molecular-based point-of-care test for chlamydia and gonorrhoea in remote Aboriginal health services in Australia. Sex Health. 2015;12:27-33.

42. Cepheid. Product information Xpert Ct/Ng. Available at: http://www.cepheid. com/us/cepheid-solutions/clinicalivd-tests/sexual-health/xpert-ct-ng.

43. Watchirs Smith LA, Hillman R, Ward J, et al. Point-of-care tests for the diagnosis of Neisseria gonorrhoeae infection: a systematic review of operational and performance characteristics. Sex Transm Infect. 2013:89:320-6.

44. Gift TL, Pate MS, Hook III EW, et al. The rapid test paradox: when fewer cases detected lead to more cases treated: a decision analysis of tests for Chlamydia trachomatis. Sex Transm Dis. 1999;26:232-40.

\section{Submit your next manuscript to BioMed Central and we will help you at every step:}

- We accept pre-submission inquiries

- Our selector tool helps you to find the most relevant journal

- We provide round the clock customer support

- Convenient online submission

- Thorough peer review

- Inclusion in PubMed and all major indexing services

- Maximum visibility for your research

Submit your manuscript at www.biomedcentral.com/submit 